\title{
Análise de tendência em séries históricas de precipitação e vazão na UGRH2 Piracicaba, Minas Gerais
}

A detecção de tendências em séries históricas de precipitação e vazão é importante para a gestão de recursos hídricos e pode ser feita por meio da utilização de testes estatísticos. Sendo assim, o objetivo do trabalho foi analisar tendências nos dados de precipitação e vazão nas estações da Unidade de Gestão de Recursos Hídricos do rio Piracicaba (UGRH2 Piracicaba), estado de Minas Gerais, utilizando os testes de Pettitt e análise de regressão linear para avaliar os dados anuais e mensais. Para as análises, foram selecionadas quinze estações pluviométricas e três estações fluviométricas, suas respectivas séries históricas foram obtidas no Portal HidroWeb da Agência Nacional de Águas. Os resultados mostraram que, para os dados de precipitação, quando o resultado dos diferentes testes foi significativo, a tendência observada foi de decréscimo para os dados anuais, enquanto que para os dados mensais os resultados variaram entre redução, com maiores ocorrências no mês de fevereiro, e elevação, com maiores ocorrências no mês de agosto. Já para a análise dos dados de vazão, quando o resultado dos diferentes testes foi significativo, a tendência observada foi de decréscimo para ambas as bases de dados, com predominância da redução da vazão no período seco. Ao avaliar o conjunto de dados, não foi possível estabelecer uma relação para os resultados obtidos entre os dados de precipitação e os dados de vazão.

Palavras-chave: Chuva; Estacionariedade; Série temporal; Teste de Pettitt; Vazão média.

\section{Trend analysis in historical series of precipitation and streamflow in the UGRH2 Piracicaba, Minas Gerais}

\begin{abstract}
Detecting trends in historical rainfall and streamflow series is important for water resource management and can be done using statistical tests. Thus, the objective of this work was to analyze trends in precipitation and streamflow data at the stations of the Piracicaba River Water Resources Management Unit (UGRH2 Piracicaba), state of Minas Gerais, using Pettitt tests and linear regression analysis to evaluate the annual and monthly data. For the analyzes, fifteen rainfall stations and three fluviometric stations were selected, their respective historical series were obtained from the HidroWeb Portal of the National Water Agency. The results showed that, for the precipitation data, when the results of the different tests were significant, the observed trend was a decrease for the annual data, while for the monthly data the results varied between reduction, with higher occurrences in the month of February, and elevation, with higher occurrences in August. For the analysis of streamflow data, when the result of the different tests was significant, the observed trend was a decrease for both databases, with predominance of reduction of streamflow in the dry period. When evaluating the data set, it was not possible to establish a relationship between the results obtained between the precipitation data and the streamflow data.
\end{abstract}

Keywords: Rain; Stationarity; Average flow; Pettitt test; Time series.

Topic: Engenharia de Recursos Hídricos

Reviewed anonymously in the process of blind peer.
Received: 09/02/2020

Approved: 09/03/2020
Micael de Souza Fraga

Instituto Mineiro de Gestão das Águas, Brasil

http://lattes.cnpq.br/5726608676680340

http://orcid.org/0000-0002-1996-9343

micaelfraga@gmail.com

Marcel Carvalho Abreu (ic

Universidade Federal Rural do Rio de Janeiro, Brasil

http://lattes.cnpq.br/1077971257668024

http://orcid.org/0000-0002-6457-421X

marcelc.abreu@gmail.com

Guilherme Barbosa Reis (it)

Universidade Federal de Viçosa, Brasil

http://lattes.cnpq.br/9426630666791133

http://orcid.org/0000-0001-7324-467X

guilherme.eaa.reis@gmail.com

\author{
Tiago Tozi iD \\ Universidade Federal de Viçosa, Brasil \\ http://lattes.cnpq.br/0777604859708404 \\ http://orcid.org/0000-0002-6585-5929 \\ tiagotozi@gmail.com \\ Sávio Augusto Rocha Pinheiro ib \\ Universidade Federal de Viçosa, Brasil \\ http://lattes.cnpq.br/4556208806020112 \\ http://orcid.org/0000-0002-7414-3747 \\ savioaugustopinheiro@gmail.com
}

Referencing this:

FRAGA, M. S.; ABREU, M. C.; REIS, G. B.; TOZI, T.; PINHEIRO, S. A. R.. Análise de tendência em séries históricas de precipitação e vazão na UGRH2 Piracicaba, Minas Gerais. Revista Ibero Americana de Ciências Ambientais, v.11, n.2, p.136-144, 2020. DOI:

http://doi.org/10.6008/CBPC2179-6858.2020.002.0016 


\section{INTRODUÇÃO}

O aumento das populações residentes nos grandes centros urbanos, assim como o aumento das atividades agropecuárias e industriais, faz com que cada vez mais os corpos hídricos sejam comprometidos, em quantidade e qualidade (SANTOS et al., 2016). Como os recursos hídricos são um bem natural importante para o desenvolvimento da sociedade, a sua falta ou escassez pode ser um grande empecilho para o desenvolvimento de uma região, fazendo com que haja a necessidade do seu monitoramento.

Dentre as variáveis utilizadas para monitorar a disponibilidade hídrica de uma determinada bacia hidrográfica ou região, destacam-se a precipitação pluviométrica e a vazão dos rios. A avaliação de ambas as variáveis em conjunto é de extrema importância, uma vez que mudanças bruscas nos regimes de precipitação implicam diretamente em mudanças nas vazões dos rios. Ferreira (2007) afirma que as vazões respondem razoavelmente à variabilidade temporal e espacial da precipitação, ou seja, grandes anomalias de precipitação quase sempre resultam em anomalias de descarga de rios.

Para apoiar o monitoramento da disponibilidade hídrica, o interesse em estudos de tendência nos dados de precipitação pluviométrica e vazão dos rios têm atraído muito a atenção dos pesquisadores. Uliana et al. (2015) relatam que tais estudos também podem ser utilizados para apoiar outros a respeito das mudanças climáticas. Para Joseph et al. (2013), a identificação de tendências sazonais contribui para a compreensão da variabilidade climática global e é essencial para o desenvolvimento de modelos hidrológicos e uma adequada gestão dos recursos hídricos. No entanto, há pouco entendimento de como os padrões espaciais e temporais das chuvas mudaram ou estão mudando.

A identificação de tendência em séries históricas pode ser feita por meio de análise estatística, sendo os testes de Pettitt (PETTITT, 1979) e de Mann-Kendall (MANN, 1945; KENDALL, 1975) os mais utilizados. Estes testes não paramétricos permitem confirmar a estacionariedade de uma série histórica e vem sendo aplicado por diversos pesquisadores em estudos hidrológicos (ALMEIDA et al., 2019; ÁVILA et al., 2014; CERA et al., 2014; FERREIRA et al., 2015; LIU et al., 2013; WANDERLEY et al., 20013).

Diante disso, o objetivo do trabalho foi analisar a tendência de séries históricas de precipitação e vazão por meio de teste estatístico não paramétrico e regressão linear em uma unidade de gestão de recursos hídricos do estado de Minas Gerais, de forma que as informações obtidas possam ser incorporadas a estudos relacionados com o planejamento e gestão de recursos hídricos na mesma unidade.

\section{METODOLOGIA}

O presente estudo foi desenvolvido na Unidade de Gestão de Recursos Hídricos do rio Piracicaba (UGRH2 Piracicaba), que, com uma área total de $5.685,86 \mathrm{~km}^{2}$, é uma das seis UGRH do rio Doce em sua porção inserida no estado de Minas Gerais (Figura 1).

Para a análise foram utilizadas séries históricas de precipitação e vazão das estações pluviométricas e fluviométricas pertencentes à Rede Hidrometeorológica da Agência Nacional de Águas (ANA). Para todas as estações foi utilizado o período máximo de dados disponíveis. Sendo assim, o período de análise foi 
diferente entre as estações pluviométricas e fluviométricas. O inventário das estações, bem como as respectivas séries históricas, é disponibilizado online no Portal HidroWeb da ANA.

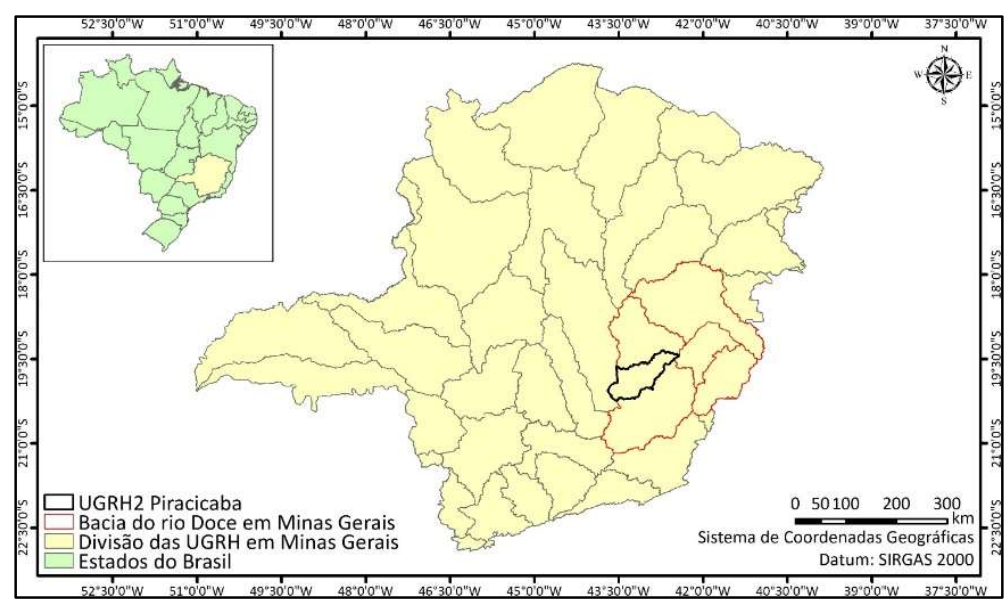

Figura 1: Localização da UGRH2 Piracicaba.

Para identificar as estações pluviométricas com influência na bacia do rio Piracicaba, foi utilizado o método do Polígono de Thiessen. A metodologia consiste em conectar as estações por trechos retilíneos, traçando linhas perpendiculares a esses trechos passando pelo meio uma linha que liga as duas estações. As linhas perpendiculares são, então, prolongadas até encontrarem as outras. O polígono é formado pela intersecção das linhas, correspondendo à área de influência de cada estação. Sendo assim, foram selecionadas todas as estações que possuíam área de influência na bacia. Já para as estações fluviométricas, foram utilizadas aquelas que se encontravam inseridas na área de estudo e que dispunham de base de dados.

Na Figura 2 são apresentadas as estações pluviométricas selecionadas pela metodologia do Polígono de Thiessen, incluindo também as estações fluviométricas que se encontram inseridas na UGRH2 Piracicaba. No total, foram selecionadas 15 estações pluviométricas (Tabela 1) e 3 estações fluviométricas (Tabela 2) para a análise de tendência.

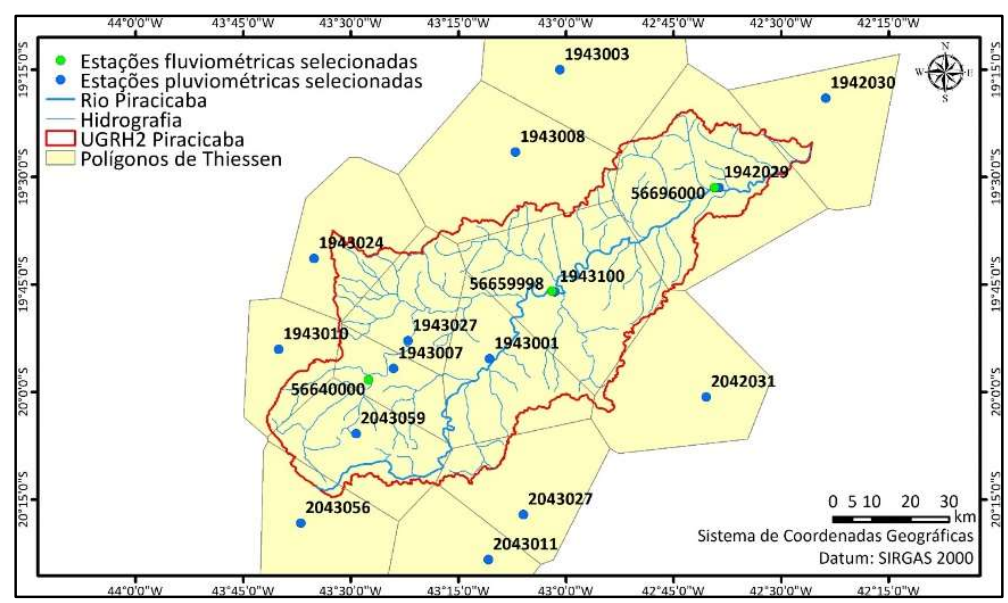

Figura 2: Resultado da seleção das estações pluviométricas e fluviométricas.

Para análise completa e confiável dos intervalos temporais estudados, foi necessário preencher as falhas das séries temporais. Para tanto, foi utilizado o método de preenchimento das falhas por regressão linear simples, conforme sugerido por Bertoni et al. (2007). Este método de preenchimento se baseia em 
dados de estações ou postos de leituras vizinhos para obtenção da correlação com a estação de interesse.

Tabela 1: Estações pluviométricas com influência na UGRH2 Piracicaba.

\begin{tabular}{|l|l|l|l|l|}
\hline Código & Nome da estação & Latitude & Longitude & Área de influência $\left(\mathrm{km}^{2}\right)$ \\
\hline 1942029 & Mário de Carvalho & $-19,525$ & $-42,644$ & 1036,42 \\
\hline 1942030 & Cenibra & $-19,316$ & $-42,396$ & 39,25 \\
\hline 1943001 & Rio Piracicaba & $-19,923$ & $-43,178$ & 839,386 \\
\hline 1943003 & Ferros & $-19,250$ & $-43,014$ & 3,47 \\
\hline 1943007 & Santa Bárbara & $-19,945$ & $-43,401$ & 409,92 \\
\hline 1943008 & Santa Maria do Itabira & $-19,442$ & $-43,118$ & 153,80 \\
\hline 1943010 & Caeté & $-19,901$ & $-43,668$ & 127,95 \\
\hline 1943024 & José de Melo & $-19,690$ & $-43,586$ & 182,45 \\
\hline 1943027 & Usina Peti & $-19,881$ & $-43,368$ & 616,86 \\
\hline 1943100 & Nova Era Telemétrica & $-19,767$ & $-43,026$ & 1376,69 \\
\hline 2042031 & Fazenda Cachoeira D'Antas & $-20,011$ & $-42,674$ & 30,83 \\
\hline 2043011 & Fazenda Paraíso & $-20,390$ & $-43,180$ & 0,35 \\
\hline 2043027 & Fazenda Ocidente & $-20,286$ & $-43,099$ & 110,93 \\
\hline 2043056 & Fazenda Água Limpa - Jusante & $-20,305$ & $-43,616$ & 44,98 \\
\hline 2043059 & Colégio Caraça & $-20,097$ & $-43,488$ & 713,464 \\
\hline
\end{tabular}

Tabela 2: Estações fluviométricas inseridas na UGRH2 Piracicaba

\begin{tabular}{|l|l|l|l|l|l|}
\hline Código & Nome da estação & Latitude & Longitude & Curso d'água & Área de drenagem $\left(\mathrm{km}^{2}\right)$ \\
\hline 56640000 & Carrapato (Brumal) & $-19,972$ & $-43,459$ & Rio Santa Bárbara & 421,30 \\
\hline 56659998 & Nova Era IV & $-19,766$ & $-43,033$ & Rio Piracicaba & 3063,10 \\
\hline 56696000 & Mário de Carvalho & $-19,524$ & $-42,655$ & Rio Piracicaba & 5269,86 \\
\hline
\end{tabular}

Para a identificação da existência de tendência significativa, ou não, nas séries históricas de precipitação e vazão, foram utilizados o teste de Pettitt e a regressão linear ao nível de $5 \%$ de significância. $O$ teste de Pettit é um teste não paramétrico que utiliza uma versão da estatística de Mann-Whitney $U_{t, N}$, a qual verifica se duas amostras $\mathrm{x}_{1}, \ldots, \mathrm{x}_{\mathrm{t}}$ e $\mathrm{x}_{\mathrm{t}+1}, \ldots, \mathrm{x}_{\mathrm{N}}$, são da mesma população ou não. A estatística de teste $U_{t, N}$ foi calculada com as Equações 1 e 2.

$$
U_{t, N}=U_{t, N}+\sum_{j=1}^{N} \operatorname{sgn}\left(x_{t}-x_{j}\right) \quad \text { Equação } 1
$$

Em que:

$$
\operatorname{sgn}=(x)\left\{\begin{array}{l}
1, \text { se } x>0 \\
0, \text { se } x=0 \\
-1, \text { se } x<0
\end{array} \quad \text { Equação } 2\right.
$$

Este teste indica o ponto em que houve uma mudança brusca na média de uma série temporal, identificando assim períodos com comportamentos distintos. A hipótese nula do teste admite ausência do ponto de mudança na série. Caso as duas amostras sejam discriminadas de populações diferentes, o teste identifica o ponto de ruptura. A estatística $\mathrm{k}(\mathrm{t})$ representa o ponto de mudança $\mathrm{t}$ significativo no qual o valor de $\left|U_{t, N}\right|$ é máximo e está associado a um nível de significância $(P)$, calculado pelas Equações 3 e 4.

$$
\begin{array}{ll}
k_{(t)}=\text { máx }_{1 \leq t \leq N}\left|U_{t, N}\right| & \text { Equação 3 } \\
P=2 e^{\left\{\frac{-6\left(K_{N}\right)^{2}}{\left(N^{3}+N^{2}\right)}\right\}} & \text { Equação 4 }
\end{array}
$$

Em que: P é o nível de significância; $K_{N}$ é o valor crítico; e N é o número de anos da série histórica. Por fim, foi realizada a análise de regressão linear entre as variáveis hidrológicas (precipitação e vazão) e os anos de análise. A análise foi utilizada para verificar tendências através da significância do coeficiente angular ( $\beta 1)$ pelo teste t de Student, que tem como hipótese nula $\left(H_{0}\right)$ que o $\beta_{1}$ é igual a zero. 
Caso a $\mathrm{H}_{0}$ seja rejeitada, admite-se a tendência e o coeficiente angular representa a taxa de acréscimo ou decréscimo anual na variável hidrológica estudada. Tanto para as estações pluviométricas, quanto para as estações fluviométricas, todas as análises foram realizadas em base anual e mensal.

\section{RESULTADOS}

Os resultados obtidos pelo teste de Pettitt e pela regressão linear, atestando a existência, ou não, de tendências significativas nos dados de precipitação total anual das estações selecionadas na UGRH2 Piracicaba, estão descritos na Tabela 3.

Tabela 3: Resultado da análise de tendência para a precipitação total anual

\begin{tabular}{|l|l|l|l|l|l|l|l|}
\hline Código & Início & \multirow{2}{*}{ Fim } & \multicolumn{2}{l|}{ Pettitt (P) } & \multicolumn{2}{l|}{ Regressão (R) } \\
\cline { 4 - 8 } & & & K & t & p-valor & $\beta_{1}$ & p-valor \\
\hline 1942029 & 1987 & 2017 & 88 & - & 0,591 & $-5,539$ & 0,407 \\
\hline 1942030 & 1987 & 2017 & 100 & - & 0,360 & $-8,721$ & 0,126 \\
\hline 1943001 & 1941 & 2017 & 312 & - & 0,912 & 0,436 & 0,824 \\
\hline 1943003 & 1942 & 2017 & 443 & - & 0,202 & $-3,309$ & 0,060 \\
\hline 1943007 & 1942 & 2017 & 360 & - & 0,539 & 0,133 & 0,951 \\
\hline 1943008 & 1942 & 2017 & 611 & 1968 & 0,013 & $-7,409$ & 0,002 \\
\hline 1943010 & 1942 & 2017 & 308 & - & 0,902 & 0,177 & 0,915 \\
\hline 1943024 & 1946 & 2016 & 216 & - & 0,537 & $-1,265$ & 0,511 \\
\hline 1943027 & 1947 & 2017 & 192 & - & 0,301 & $-1,373$ & 0,507 \\
\hline 1943100 & 2003 & 2017 & 38 & - & 0,118 & $-27,188$ & 0,130 \\
\hline 2042031 & 1982 & 2017 & 84 & - & 0,735 & $-3,545$ & 0,514 \\
\hline 2043011 & 1942 & 2017 & 297 & - & 0,985 & $-0,334$ & 0,868 \\
\hline 2043027 & 1969 & 2017 & 100 & - & 0,195 & 0,809 & 0,823 \\
\hline 2043056 & 1985 & 2017 & 108 & - & 0,380 & $-9,944$ & 0,032 \\
\hline 2043059 & 1983 & 2017 & 146 & - & 0,135 & $-23,319$ & 0,003 \\
\hline
\end{tabular}

Na Tabela 4 são apresentados os resultados finais da análise de tendência para a precipitação mensal referente aos testes utilizados, sendo eles interpretados da seguinte maneira: tendência significativa de elevação ( $\uparrow)$, tendência significativa de redução $(\downarrow)$; e sem tendência (-).

Tabela 4: Resultado da análise de tendência para a precipitação total mensal.

\begin{tabular}{|c|c|c|c|c|c|c|c|c|c|c|c|c|c|c|c|c|c|c|c|c|c|c|c|c|}
\hline Mês & Jar & & Fel & & $M$ & & $A b$ & & $\mathrm{Ma}$ & & Jur & & Jul. & & $\mathrm{Ag}$ & & Set & & $\mathrm{Ou}$ & & Nol & & $\mathrm{De}$ & \\
\hline Código & $P$ & $\mathrm{R}$ & $P$ & $\mathrm{R}$ & $P$ & $\mathrm{R}$ & $P$ & $\mathrm{R}$ & $P$ & $\mathrm{R}$ & $P$ & $\mathrm{R}$ & $P$ & $\mathrm{R}$ & $\mathrm{P}$ & $\mathrm{R}$ & $P$ & $\mathrm{R}$ & $P$ & $\mathrm{R}$ & $P$ & $\mathrm{R}$ & $P$ & $\mathrm{R}$ \\
\hline 1942029 & - & - & - & - & - & - & - & 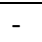 & - & - & - & 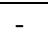 & - & - & - & - & - & - & - & - & $\downarrow$ & - & - & - \\
\hline 1942030 & - & - & - & - & - & - & - & - & - & - & - & - & $\downarrow$ & $\downarrow$ & - & - & - & - & - & - & - & - & - & - \\
\hline 1943001 & - & - & - & - & - & - & - & - & - & - & - & - & - & - & $\uparrow$ & - & - & - & - & - & - & - & - & - \\
\hline 1943003 & $\downarrow$ & - & - & $\downarrow$ & - & - & - & - & - & - & - & - & - & - & - & - & - & - & - & $\downarrow$ & - & - & - & - \\
\hline 1943007 & - & - & - & - & - & - & - & - & - & - & - & - & - & - & $\uparrow$ & - & - & - & - & - & $\uparrow$ & - & - & - \\
\hline 1943008 & - & - & $\downarrow$ & $\downarrow$ & - & - & - & - & - & - & - & - & - & - & - & - & - & - & - & - & - & - & $\downarrow$ & $\downarrow$ \\
\hline 1943010 & - & - & - & - & - & - & - & - & $\uparrow$ & - & - & - & $\uparrow$ & $\uparrow$ & $\uparrow$ & - & - & - & - & - & - & - & - & - \\
\hline 1943024 & - & - & - & $\downarrow$ & - & - & - & - & - & - & - & - & - & - & $\uparrow$ & - & - & - & - & - & - & - & - & - \\
\hline 1943027 & - & - & - & $\downarrow$ & - & - & - & - & - & - & - & - & - & - & - & - & - & - & - & $\downarrow$ & - & - & - & - \\
\hline 1943100 & - & - & - & - & - & - & - & - & - & - & - & - & - & - & - & - & $\downarrow$ & - & - & - & - & - & - & - \\
\hline 2042031 & - & - & - & - & - & - & - & - & - & - & - & - & - & - & - & $\downarrow$ & - & - & - & - & - & - & - & - \\
\hline 2043011 & - & - & - & - & - & - & - & - & - & - & - & - & - & - & - & - & $\uparrow$ & - & - & - & - & - & - & - \\
\hline 2043027 & - & - & - & - & - & - & - & - & - & - & - & - & - & - & - & - & - & - & - & - & - & - & - & - \\
\hline 2043056 & - & - & - & - & - & - & - & - & - & - & $\uparrow$ & $\uparrow$ & - & - & - & - & 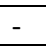 & - & - & - & - & - & - & - \\
\hline 2043059 & - & - & - & - & - & - & - & - & - & - & - & - & - & - & - & - & - & - & - & - & - & - & - & $\downarrow$ \\
\hline
\end{tabular}

Nas Tabelas 5 e 6 são apresentados os resultados de tendência para a vazão média anual e vazão média mensal, respectivamente. Ressalta-se que a apresentação dos resultados segue a mesma lógica do apresentado para os dados de precipitação total anual e precipitação mensal. 
Tabela 5: Resultado da análise de tendência para a vazão média anual

\begin{tabular}{|l|l|l|l|l|l|l|l|}
\hline Código & Início & \multirow{2}{*}{ Fim } & Pettitt & Regressão \\
\cline { 4 - 8 } & & & K & t & p-valor & $\beta_{1}$ & p-valor \\
\hline 56640000 & 1955 & 2015 & 306 & - & 0,252 & 0.000 & 0.993 \\
\hline 56659998 & 1990 & 2017 & 99 & - & 0,171 & -0.727 & 0.044 \\
\hline 56696000 & 1987 & 2017 & 112 & - & 0,202 & -1.044 & 0.595 \\
\hline
\end{tabular}

Tabela 6: Resultado da análise de tendência para a vazão média mensal

\begin{tabular}{|c|c|c|c|c|c|c|c|c|c|c|c|c|c|c|c|c|c|c|c|c|c|c|c|c|}
\hline Mês & \multicolumn{2}{|c|}{ Jan. } & \multicolumn{2}{|c|}{ Fev. } & \multicolumn{2}{|c|}{ Mar. } & \multicolumn{2}{|c|}{ Abr. } & \multicolumn{2}{|c|}{ Mai. } & \multicolumn{2}{|c|}{ Jun. } & \multicolumn{2}{|c|}{ Jul. } & \multicolumn{2}{|c|}{ Ago. } & \multicolumn{2}{|c|}{ Set. } & \multicolumn{2}{|c|}{ Out. } & \multicolumn{2}{|c|}{ Nov. } & \multicolumn{2}{|c|}{ Dez. } \\
\hline Código & $\mathrm{P}$ & $R$ & $\mathrm{P}$ & $R$ & $\mathrm{P}$ & $\mathrm{R}$ & $\mathrm{P}$ & $R$ & $P$ & $\mathrm{R}$ & $\mathrm{P}$ & $R$ & $P$ & $R$ & $P$ & $R$ & $\mathrm{P}$ & $\mathrm{R}$ & $\mathrm{P}$ & $\mathrm{R}$ & $\mathrm{P}$ & $R$ & $P$ & $R$ \\
\hline 56640000 & - & - & - & - & - & - & - & - & - & - & $\downarrow$ & - & $\downarrow$ & - & $\downarrow$ & 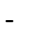 & $\downarrow$ & - & $\downarrow$ & - & - & - & - & - \\
\hline 56659998 & - & - & $\downarrow$ & - & - & - & - & - & - & - & - & - & - & - & - & $\downarrow$ & $\downarrow$ & $\downarrow$ & - & - & - & - & - & - \\
\hline 56696000 & - & - & $\downarrow$ & - & - & - & - & - & - & - & - & - & - & - & 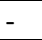 & - & - & - & - & - & - & - & - & - \\
\hline
\end{tabular}

\section{DISCUSSÃO}

Pelos resultados da análise de tendência para a precipitação total anual apresentados na Tabela 3, foram observadas discrepâncias entre os testes, o qual o teste de Pettitt indicou tendência significativa em uma estação (1943008), enquanto a análise do coeficiente angular da regressão entre a precipitação e os anos de observação indicou coeficiente angular diferente de zero em 3 estações (1943008, 2043056 e 2043059). Porém, quando o resultado dos diferentes testes foi significativo, a tendência observada foi de decréscimo da precipitação anual. A estação Santa Maria do Itabira (código 1943008) apresentou tendência significativa ( $p$-valor $<0,05$ ) nos dois testes, com propensão à redução nos dados de chuva anual de até 7,4 mm por ano. O ponto de mudança ocorreu no ano de 1968. Entretanto, ao avaliar a Figura 1 e a Tabela 1 , verifica-se que a estação possui apenas 153,80 km² de influência na UGRH2 Piracicaba. Na estação Colégio Caraça (2043059), apesar da tendência pelo teste de Pettitt não ter sido significativa, foi observado o maior coeficiente angular significativo, indicando decréscimo anual de até 23,3 mm de chuva.

Ao avaliar os resultados da análise de tendência para a precipitação mensal (Tabela 4), é possível verificar uma maior ocorrência das tendências significativas nos meses de fevereiro (tendências de redução) e agosto (tendências de elevação). Para os demais meses, os resultados foram isolados entre as estações, com discrepância entre os testes aplicados e variando igualmente entre redução e elevação.

Além da tendência significativa de redução para a precipitação total anual, a estação Santa Maria do Itabira (código 1943008) também apresentou tendência significativa de redução da precipitação por ambos os testes para os meses de fevereiro e dezembro, que são considerados meses do período chuvoso na UGRG2 Piracicaba. Sendo assim, pode-se concluir que a redução do total precipitado ocorrida nesses meses pode ter contribuído para o resultado encontrado na análise do total anual para a estação em questão, uma vez que redução em meses de maior total precipitado possuem maior influência na análise.

No Brasil, foram realizadas análises de tendência em poucos estados, como em São Paulo, Minas Gerais, Bahia, Espírito Santo e Mato Grosso. Com exceção das análises realizadas por Groppo et al. (2005), que encontraram tendência significativa para a precipitação, nos demais trabalhos o predomínio dos resultados também foi de ausência de tendência significativa nos dados (ALEXANDRE et al., 2010; BLAIN, 2010; LIMA et al., 2011; MARENGO et al., 2005; MESCHIATTI et al., 2012; NATIVIDADE et al., 2017; ROSSIN et al., 2015; ULIANA et al., 2015). 
Em Minas Gerais, Santos et al. (2017) e Natividade et al. (2017) detectaram tendências heterogêneas em relação à precipitação. Enquanto que nas regiões Norte e Leste do estado, as chuvas tendem a diminuir, nas regiões Centro e Sul do estado as chuvas tendem a aumentar. Esses resultados são contrários aos encontrados neste estudo, que detectou estações pluviométricas com tendências de decréscimo de precipitação, especialmente em fevereiro (estação chuvosa).

Ressalta-se que em termos de mudanças climáticas, a precipitação é a variável meteorológica com maior incerteza na análise, o que justifica a complexidade e as diferenças nos resultados encontrados em diversos estudos com propósitos similares. Ainda, é importante destacar que a deteç̧ão de tendências significativas através de testes estatísticos não pode ser atribuída às mudanças climáticas dada a complexidade da dissociação dessas mudanças às variações naturais do clima, assim como creditar as tendências encontradas como consequências de ações antrópicas, uma vez que o regime de chuvas não pode ser atribuído somente às mudanças locais (GROPPO et al., 2005).

Ao analisar a vazão média anual (Tabela 5), nota-se que teste de Pettitt não detectou pontos de ruptura nas séries de vazões anuais, enquanto que a análise de regressão linear detectou tendência significativa de redução na vazão média anual da estação 56659998, em taxa de decréscimo de aproximadamente $0,73 \mathrm{~m}^{3} \mathrm{~s}^{-1}$. Já para a análise mensal (Tabela 6), o teste de Pettitt apresentou maior número de tendências significativas em relação a análise de regressão linear.

Pelo teste de Pettitt, a estação de Carrapato (Brumal) (código 56640000) apresentou tendência significativa de redução entre os meses junho e outubro, que correspondem ao período seco da UGRH2 Piracicaba. A análise de regressão linear também detectou tendências de redução das vazões nos meses de estiagem dessa estação. A estação Nova Era IV (código 56659998) apresentou tendências de decréscimo das vazões entre os meses de agosto e setembro, também período de estiagem na região. Apesar da tendência significativa de redução em meses consecutivos do período seco, a redução não foi evidente o suficiente para provocar redução significativa nos dados anuais, o que reforça a ideia de que os resultados do período chuvoso têm mais influência do que o período seco quando se avalia os dados anuais.

Ao confrontar os resultados das análises de tendência mensal dos dados de precipitação (Tabela 4) com os dados de vazão (Tabela 6), não foi possível encontrar uma relação entre os mesmos, ou seja, as tendências detectadas não podem ser explicadas suficientemente pela relação chuva-vazão. Tal fato pode ser exemplificado pela estação fluviométrica Carrapato (Brumal) (código 56640000), localizada na cabeceira da UGRH2 Piracicaba, que, apesar de ter apresentado diversos meses com tendência significativa de redução (junho a outubro), não foi constatada a mesma tendência nos dados de precipitação das estações pluviométricas ao seu entorno, sendo elas: Colégio Caraça (código 2043059), Santa Bárbara (código 1943007) e Usina Peti (1943027). Kliment et al. (2011), em um estudo realizado na República Checa, também detectaram tendência de decréscimo na vazão sem conexão com decréscimo de precipitação.

Levando em conta os resultados encontrados, destaca-se que as vazões são influenciadas não somente pelo clima, mas também pelo uso e ocupação do solo (ULIANA et al., 2014), sendo mais facilmente influenciada em termos locais em relação a precipitação. A tendência de decréscimo pode estar associada a 
menor infiltração da água no solo, favorecendo maior escoamento superficial e menor recarga do lençol freático que iria contribuir com o aumento das vazões no período de estiagem (ULIANA et al., 2014).

\section{CONCLUSÕES}

Os testes de tendência possibilitaram a identificação de tendências significativas nas séries históricas de precipitação e vazão anuais, bem como nas séries de precipitação e vazão mensais. Para os dados de precipitação, quando o resultado dos diferentes testes foi significativo, a tendência observada foi de decréscimo para os dados anuais e variando entre redução e elevação para os dados mensais. Já para os dados de vazão, a tendência observada foi apenas de decréscimo.

Para os resultados das análises dos dados anuais, foi possível inferir que as tendências significativas ocorridas no período chuvoso possuem maior influência do que as ocorridas no período seco. Não foi possível perceber uma relação entre os resultados obtidos paras as tendências dos dados de precipitação com os dados de vazão, inferindo que os resultados de tendência significativa de redução obtidos para os dados de vazão mensal estejam associados a outros fatores que não seja a precipitação.

\section{REFERÊNCIAS}

ALEXANDRE, G. R.; BAPTISTA, M. B.; NAGHETTINI, M.. Estudo para identificação de tendências do regime pluvial na região metropolitana de Belo Horizonte a partir de métodos estatísticos. Revista Brasileira de Recursos Hídricos, v.15, n.2, p.115-126, 2010.

ALMEIDA, L. T.; SILVA, F. B.; CECÍLIO, R. A.; ABREU, M. C.; FRAGA, M. S.. Análise do comportamento da vazão e precipitação na influência de enchentes na bacia hidrográfica a montante da cidade de Itajubá. Revista Augustus, v.24, n.49, p.124-145, 2019.

ÁVILA, L. F.; MELLO, C. R.; YANAGI, S. N. M.; SACRAMENTO NETO, O. B.. Tendências de temperaturas mínimas e máximas do ar no Estado de Minas Gerais. Pesquisa Agropecuária Brasileira, v.49, n.4, p.247-256, 2014.

BERTONI, J. C.; TUCCI, C. E. M.. Precipitação. In: TUCCI, C. E. M.. Hidrologia: Ciência e Aplicação. 4 ed. Porto Alegre: ABRH, 2009.

BLAIN, G. C.. Tendências e variações climáticas em séries anuais de precipitação pluvial do Estado de São Paulo. Bragantia, v.69, n.3, p.765-770, 2010.

CERA, J. C.; FERRAZ, S. E. T.. Variações climáticas na precipitação no sul do Brasil no clima presente de futuro. Revista Brasileira de Meteorologia, v.30, n.1, p.81-88, 2014.

FERREIRA, V. O.. Paisagem, recursos hídricos e desenvolvimento econômico na Bacia do Rio Jequitinhonha, em Minas Gerais. Tese (Doutorado em Geografia) - Universidade Federal de Minas Gerais, Belo Horizonte, 2007.

FERREIRA, D. H. L.; PENEREIRO, J. C.; FONTOLAN, M. R. Análises estatísticas de tendências das séries hidroclimáticas e de ações antrópicas ao longo das sub-bacias do rio Tietê. HOLOS, v.2, p.50-68, 2015.

GROPPO, J. D.; MORAES, J. M.; BEDUSCHI, C. E.; MARTINELLI, L. A.. Análise de séries temporais de vazão e precipitação em algumas bacias do Estado de São Paulo com diferentes graus de intervenções antrópicas. Revista Geociências, v.24, n.2, p.181-193, 2005.

JOSEPH, J. F.; FALCON, H. E.; SHARIF, H. O.. Hydrologic Trends and Correlations in South Texas River Basins: 19502009. Journal of Hydrologic Engineering, v.18, n.2, p.16531662, 2013.

KENDALL, M. G.. Rank Correlation Methods. 4 ed. Londres: Charles Griffin, 1975.

KLIMENT, Z.; MATOUŠKOVÁ, M.; LEDVINKA, O.; KRÁLOVEC, V.. Trend analysis of rainfall-runoff regimes in selected headwater areas of the Czech Republic. Journal of Hydrology and Hydromechanics, v.59, n.1, p.36-50, 2011.

LIMA, J. R. A.; NEVES, D. J. D.; ARAÚJO, L. E.; AZEVEDO, P. V.. Identificação de tendências climáticas no Estado da Bahia. Revista de Geografia, v.28, n.3, p.172-187, 2011.

LIU, X.; DAI, X.; ZHONG, Y.; LI, J.; WANG, P.. Analysis of changes in the relationship between precipitation and streamflow in the Yiluo River, China. Theoretical and Applied Climatology, v.114, p.183-191, 2013.

MANN, H. B.. Nonparametric tests against trend. Econometrica, v.13, n.3, p.245-259, 1945.

MARENGO, J. A.; ALVES, L. M.. Tendências hidrológicas da Bacia do Rio Paraíba do Sul. Revista Brasileira de Meteorologia, v.20, n.2, p.215-226, 2005. 
MESCHIATTI, M. C.; FONTOLAN, M. R.; PENEREIRO, J. C.; FERREIRA, D. H. L.. Caracterização estatística de tendências em séries anuais de dados hidroclimáticos no Estado de São Paulo. Revista Geográfica Acadêmica, v.6, n.1, p.52-64, 2012.

NATIVIDADE, U. A.; GARCIA, S. R.; TORRES, R. R.. Tendência dos Índices de Extremos Climáticos Observados e Projetados no Estado de Minas Gerais. Revista Brasileira de Meteorologia, v.32, n.4, p.600-614, 2017.

PETTITT, A. N.. A Non-Parametric Approach to the ChangePoint Problem. Applied Statistics, v.28, p.126-135, 1979.

ROSSIN, C.; AMORIM, R. S. S.; MORAIS, T. S. T.. Análise de tendências hidrológicas na bacia do rio das Mortes. Revista Brasileira de Recursos Hídricos, v.20, n.4, p.991-998, 2015.
SANTOS, D. F.; MARTINS, F. B.; TORRES, R. R.. Impacts of climate projections on water balance and implications on olive crop in Minas Gerais. Revista Brasileira de Engenharia Agrícola e Ambiental, v.21, n.2, p.77-82, 2017.

SANTOS, V. O.; NISHIYAMA, L.. Tendências hidrológicas no alto curso da bacia hidrográfica do rio Uberaba, Minas Gerais. Caminhos de Geografia, v.17, n.58, p.196-212, 2016

ULIANA, E. M.; SILVA, D. D.; ULIANA, E. M.; RODRIGUES, B. S.; CORRÊDO, L. P.. Análise de tendência em séries históricas de vazão e precipitação: uso de teste estatístico não paramétrico. Ambiente \& Água, v.10, n.1, p.82-88, 2015.

WANDERLEY, H. S.; SEDIYAMA, G. C.; JUSTINO, F. B.; ALENCAR, L. P.; DELGADO, R. C.. Variabilidade de precipitação no sertão do São Francisco, estado de Alagoas. Revista Brasileira de Engenharia Agrícola e Ambiental, v.17, n.7, p.790-795, 2013.

A CBPC - Companhia Brasileira de Produção Científica (CNPJ: 11.221.422/0001-03) detém os direitos materiais desta publicação. Os direitos referem-se à publicação do trabalho em qualquer parte do mundo, incluindo os direitos às renovações, expansões e disseminações da contribuição, bem como outros direitos subsidiários. Todos os trabalhos publicados eletronicamente poderão posteriormente ser publicados em coletâneas impressas sob coordenação da Sustenere Publishing, da Companhia Brasileira de Produção Científica e seus parceiros autorizados. Os (as) autores (as) preservam os direitos autorais, mas não têm permissão para a publicação da contribuição em outro meio, impresso ou digital, em português ou em tradução. 